\title{
THE PRESENT STATUS OF ASTROMETRY AT OPTICAL AND RADIO WAVELENGTHS
}

\author{
K. J. JOHNSTON \\ Remote Sensing Division \\ Naval Research Laboratory \\ Washington, D.C. 20375, USA
}

\begin{abstract}
A Review is presented of the present status of astrometry towards defining a radio/optical reference frame.
\end{abstract}

\section{Introduction}

Over the past twenty five years the development of astrometry at radio wavelengths using very high angular resolution instruments such as interferometers has led to improving the accuracy of the determination of the positions of celestial objects from 100 to less than 1 milliarcsecond. The use of these interferometers for wide angle astrometry defines a radio/optical reference frame. In this context, wide angle is defined as determining the relative positions of celestial objects separated by angles greater than 5 degrees. Over small angles ( $<1$ degree) relative positional accuracies can be achieved to an order of magnitude better than over wide angles. At radio wavelengths the highest accuracy for astrometric measurements has been achieved using Very Long Baseline Interferometry (VLBI), while optical wavelength measurements have been made using single aperture instruments i.e. meridian transit circles and astrolabes. There has recently been the development of optical interferometry for astrometry.

The major astronomical impetus in establishing a global reference frame is to determine the motions of celestial objects, obtain improved values for astronomical constants, and overlay optical and radio emission from celestial objects. Here in the context of this meeting, we are concerned with the accuracies with which one can measure the positions of celestial objects at a single wavelength range and at multiple wavelengths so that optical and radio emission can be compared. At this time, this can be accomplished to accuracies of order 100 and 1 milliarcsecond at optical and radio wavelengths respectively. However, recent developments in astrometry should allow optical accuracies to approach 0.003 arcseconds by 1995 . With this increased accuracy, important astrophysical studies can be made by comparing the spatial coincidence of the optical and radio emission for objects such as SN 1987A, the circumstellar envelopes of evolved stars, starburst/Seyfert galaxies and galactic obscured regions such as the galactic center.

\section{Reference Frames}

Reference frames are defined by the positions of the objects making up the frame. The radio reference frame is defined by the positions of the extragalactic sources (Ma et al. 1990). These objects are intense radio sources but are visual magnitude $13^{\text {th }}$ or fainter with the majority being $18^{\text {th }}$ magnitude or fainter. The optical reference frame, the FK5, is defined by the positions of bright stars. The accuracy 
of the FK5 positions is of order 50 milliarcseconds over the globe (Fricke et al. 1988). Direct measurement of the optical positions of extragalactic radio sources to relatively nearby faint stars and relating the positions of these faint stars to FK5 stars has shown that the frames can be aligned to approximately 100 milliarcseconds. This is the level of accuracy of the FK5 frame. If the HIPPARCOS mission is successful, the accuracy of the optical reference frame will approach a few milliarcseconds. Using the technique previously mentioned using HIPPARCOS stars should result in accuracy of order 10 milliarcseconds. In order to relate the reference frames at higher accuracies, objects which emit optical emission as bright as the FK5 stars must be used. Bright stars which emit observable radio emission such as continuum emission from variables or maser emission from $\mathrm{SiO}$ and $\mathrm{H}_{2} \mathrm{O}$ in circumstellar envelopes of late type stars are candidates. Further the relative positions of star/quasar pairs measured by space telescope could be used. Using these objects, the frames may be related at accuracies as small as 3 millarcseconds. This assumes that the accuracy of the optical reference frame will approach these accuracies. Further the parallax, proper motion and stellar orbits, if the reference stars are binaries, must be determined to these accuracies.

\section{Present Developments Towards a Radio/Optical Reference Frame}

The IAU Working Group on the Radio/Optical Reference Frame, formed by Commission 24 in 1978 had as its charter to draw up a list of suitable candidates for a radio/optical reference frame. A candidate list of 234 sources was made with their radio positions based on a weighted mean by error of 9 radio catalogs and had an average accuracy better than 0.01 arcsec (Argue et al. 1984). One of the major shortcomings of this work was the paucity of sources in the southern hemisphere.

A program to establish a radio/optical reference frame was undertaken in 1987 and is described in IAU Symposium 110 (Johnston et al. 1988). This program has as its goal the establishment of a reference frame based on the radio/optical positions of 400 sources uniformly distributed globally over the celestial sphere. These sources are to be compact in their radio/optical emission. They should be brighter than visual magnitude 19 and have a total flux density greater than $1 \mathrm{Jy}$ at $5 \mathrm{GHz}$. The method of construction of the reference frame is to adopt the radio positions as the reference positions of the frame and upgrade the optical frame by the adopting the radio positions. Recent results of this program are described by Reynolds et al.(1993) in this volume. The accuracy of the radio source positions in the northern and southern hemispheres should approach 0.5 and 1 milliarcsecond respectively. The optical positions will be accurate to 50 milliarcseconds on the FK5 frame.

This program will place all data in a uniform data base which will be used to obtain a global solution from first principles. This data base will be updated as future observations are made. This will be accomplished for the radio observations. The optical data will also be maintained in a data base. The initial relationship between the reference frames will be at the 50 milliarcsecond level (Johnston et al. 1991). 


\section{Future Developments}

There are several areas which will contribute to the refinement of the reference frame. Development of radio astronomy facilities for astrometry such as the VLBA, and improvements to MERLIN, the addition of many facilities to VLB networks for astronomy and geodesy will add to the accuracy of the radio positions of extragalactic sources. Optical astrometry will show a drastic improvement with the HIPPARCOS catalog which will appear two years after the completion of observations. This catalog will most likely appear in 1995/1996. By this time, observations of close star/quasar pairs with the Hubble Space Telescope may be available. Astrometric optical interferometric measurements may also contribute to improvements in aligning the frames. An instrument is presently under construction at Flagstaff Arizona by the United States Naval Observatory and the Naval Research Laboratory. (See the papers by Hutter and Simon in this volume).

\section{Acknowledgements}

K.J.J. thanks Gart Westerhout for presenting this paper at IAU Symposium 158

\section{References}

Argue, A. N., deVegt, C., Elsmore, B., Fanselow, J., Harrington, R., Hemenway, P., Johnston, K.J., Kuhr, H., Kumkova, I., Neil, A.E., Walter, H., Witzel, A. 1984 Astronomy and Astrophysics 130, 191-192.

Fricke, W., Schwan, H., Lederle, T., Bastian, U., Bien, R., Burkhardt, G., du Mont, B., Hering, R., Jährling, R., Jahrei $\beta$, H., Röser, S., Schwerdtfeger, H.M., and Walter, H.G. 1988, Fifth Fundamental Catalogue (Veröffentlichungen Astronomisches Recheninstitut, Heidelberg).

Johnston, K. J., Russell, J.L., deVegt, C., Hughes, J., Jauncey, D., White, G., Nicolson, G. 1988 in, The Impact of VLBI on Astrophysics and Geophysics, IAU Symposium 129, eds., Reid, M. and Moran, J., Reidel, Dordrecht 317.

Johnston, K. J., Russell, J.L., deVegt, C., Zacharias, N., Hindsley, R., Hughes, J., Jauncey, D., Reynolds, J.E., Nicholson, J.E., Ma, C. 1991 in, Reference Systems, IAU Colloquium 127, eds., Hughes, J., Smith, C., and Kaplan, G., United States Naval Observatory, Washington 123.

Ma, C., Shaffer, D.B., deVegt, C., Johnston, K.J., Russell, J.L. 1990 Astronomical Journal 99, 1284 .

\section{Discussion:}

Burke:

To what extent can radio measurements of the classical solar-system based coordinate system replace the optical methods? Could both the earth-based and extragalactic frames be tied together with greater precision that way?

Westerhout:

The classical "fundamental coordinate system", based on the solar system, will be replaced by an extragalactic reference system, per IAU resolution. Dr Chr. de Vegt is chairman of the IAU working group that is to achieve this. It is expected that solar system observations by radio and radar will eventually tie the solar system frame to the extragalactic reference frame. The earth-based system is already almost tied to the extragalactic system. Precession and nutation are being derived. Earth orientation is entirely based on the extragalactic (IERS) reference frame now! 


\section{Baldwin:}

You mentioned the contribution that Hipparcos will make in this programme. Isn't it the case that it is a huge perturbation on the subject which requires a fundamental change in the way the programme should go ahead in the future?

Westerhout:

That is correct. Hipparcos will have a major impact at the time it is released, but unless it is maintained, the Hipparcos reference frame will degenerate rapidly. And in any case, Hipparcos still must be tied to the extragalactic frame. It seems likely that the FK5 reference frame will be attached to the extragalactic frame with major help from Hipparcos.

\section{Davies:}

When tying together the optical and radio frames it is important to understand the structure of the objects which may be different at the two wavelengths. For example, the stellar objects selected for study at radio wavelengths are often active objects with complex structures which are not repeated in the optical. A high resolution, high surface brightness array like MERLIN can identify the free-free emission directly associated with the optical object.

Westerhout:

The understanding of the astrophysics of the reference frame objects is essential for the realization of that frame, and the MERLIN contribution to the star positions and circumstellar structure will be essential.

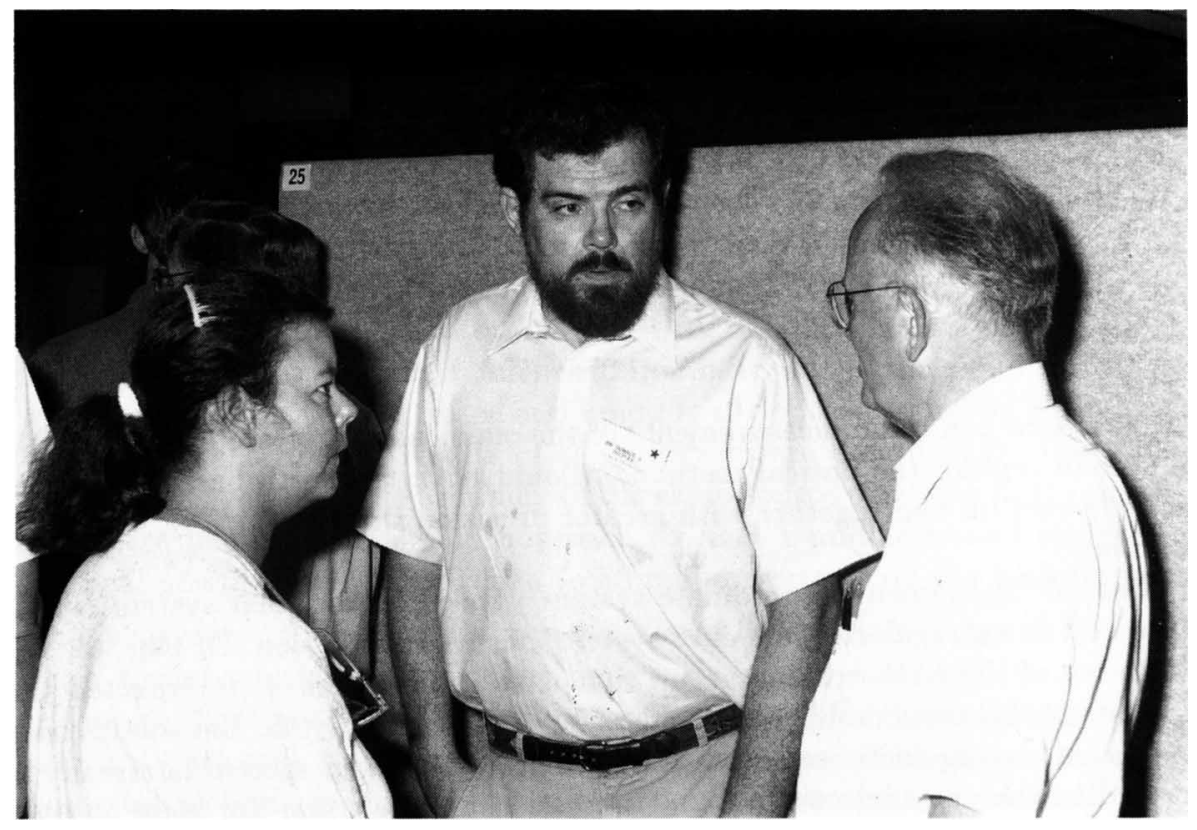

